\title{
Avaliação do Programa Jovem Aprendiz a partir de um Estudo Quase-Experimental
}

\author{
Maria da Conceição Oliveira Villar ${ }^{1}$ \\ Orcid.org/0000-0003-3146-2876 \\ Luciana Mourão*, 1 \\ Orcid.org/0000-0002-8230-3763
}

${ }^{1}$ Universidade Salgado de Oliveira, Niterói, RJ, Brasil

\section{Resumo}

No Brasil a mão de obra juvenil apresenta uma relação exploratória e sem foco na qualidade da formação profissional. Esta pesquisa objetivou avaliar a contribuição do Programa Jovem Aprendiz na vida de adolescentes de 15 a 18 anos, em termos de desenvolvimento profissional, empregabilidade e autoeficácia. Foi realizado um estudo quase-experimental, com dois momentos de coleta de dados - no início da formação e seis meses depois. Foram pesquisados 250 adolescentes participantes do programa (grupo experimental) e 259 não participantes (grupo controle). As escalas de desenvolvimento profissional, empregabilidade e autoeficácia apresentavam evidências de validade psicométrica. Para comparar os grupos de pesquisa, utilizou-se a ANOVA mista 2 (grupo) X 2 (tempo) com medidas repetidas (intra-sujeitos). Os resultados indicaram que o programa favorece o desenvolvimento profissional, a empregabilidade e a autoeficácia. São apresentadas sugestões para melhoria do Programa Jovem Aprendiz e reflexões para as políticas públicas voltadas para a formação profissional e para o Ensino Médio.

Palavras-chave: Jovem aprendiz, formação profissional, desenvolvimento profissional, empregabilidade, autoeficácia.

\section{Evaluation of the Young Apprentice Program through a Quasi-Experimental Study}

\begin{abstract}
In Brazil, the youth workforce presents an exploratory relationship, without focus on the quality of professional training. This study aimed to evaluate the contribution of the Young Apprentice Program in the life of adolescents, 15 to 18 years of age, in terms of professional development, employability, and self-efficacy. A quasi-experimental study was carried out, with two data collection moments - at the start of the training and six months later. A total of 250 adolescents from the program (experimental group) and 259 non-participants (control group) were studied. The professional development, employability,
\end{abstract}

* Endereço para correspondência: Universidade Salgado de Oliveira, Programa de Pós-Graduação em Psicologia, Rua Marechal Deodoro, 217, Bloco A, $2^{\circ}$ andar, Niterói, RJ, Brasil 24030-060. Fone: (21) 98886-6764. E-mail: ceissavillar@yahoo.com.br e mourao.luciana@gmail.com

Esta pesquisa contou com financiamento da Coordenação de Aperfeiçoamento de Pessoal de Nível Superior CAPES. 
and self-efficacy scales presented evidence of psychometric validity. To compare the research groups, mixed ANOVA, 2 (group) X 2 (time), with repeated measures (intra-subject), was used. The results indicated that the program aids professional development, employability and self-efficacy. Suggestions for improving the program and reflections regarding public policies aimed at High School Education are presented.

Keywords: Young apprentice, professional training, professional development, employability, selfefficacy.

\section{Evaluación del Programa Joven Aprendiz a partir de un Estudio Casi-Experimental}

\section{Resumen}

En Brasil la mano de obra juvenil tiene una relación de explotación y no enfoca la calidad de la formación profesional. Esta investigación objetivó evaluar la contribución del Programa Joven Aprendiz en la vida de adolescentes de 15 a 18 años, en los términos del Desarrollo Profesional, Empleabilidad y Autoeficacia. Se realizó un estudio casi-experimental, con dos momentos de recolección de datos - al inicio de la formación y seis meses después. Se investigaron a 250 adolescentes participantes del programa (grupo experimental) y 259 no participantes (grupo control). Las escalas de desarrollo profesional, empleabilidad y autoeficacia presentaban evidencias de validad psicométrica. Para comparar los grupos de estudio, se utilizó la ANOVA mixta 2 (grupo) X 2 (tiempo) con medidas repetidas (intra-sujetos). Los resultados indicaron que el programa favorece el desarrollo profesional, la empleabilidad y la autoeficacia. Se presentan sugestiones para mejorar el Programa y reflexiones dirigidas a las políticas públicas de la Enseñanza Secundaria.

Palabras clave: Joven aprendiz, formación professional, desarrollo professional, empleabilidad, autoeficacia.

Cerca de $30 \%$ da população mundial é de adolescentes e aproximadamente $80 \%$ vivem em países em desenvolvimento - esses números sinalizam para a importância de compreender aspectos relativos a esse público-alvo, entre eles a educação e os desafios para a prática profissional (Cerqueira-Santos, Melo, \& Koller, 2014). Especificamente no que diz respeito à participação do adolescente no mercado de trabalho, observa-se uma elevada participação no Brasil quando comparada a outros países, o que decorre tanto da abundância desse público, quanto do caráter exploratório ainda existente sobre o mesmo (Oliveira, 2009).

O uso inadequado da mão de obra infanto-juvenil não pode ser desconsiderado, pois é um fenômeno que persiste mesmo mediante dos preceitos legais que regularizam o assunto - dados do Instituto Brasileiro de Geografia e Estatística (IBGE) apontam dois milhões de brasileiros en- tre 14 e 17 anos trabalhando de forma irregular (2016). Apenas a metade dos jovens de 18 a 24 anos conclui o Ensino Médio (IBGE, 2013) e há elevada distorção de idade-série com significativa parcela de estudantes na faixa etária de 18 a 19 anos. Além disso, 49\% dos jovens de 15 a 17 anos estão fora do Ensino Médio e 18\% fora das escolas (Schwartzman \& Castro, 2013).

De fato, em pesquisas nacionais e estrangeiras, o trabalho de adolescentes apresenta uma relação direta com o nível socioeconômico e com o abandono ou desempenho escolar. As pesquisas apontam que enquanto o trabalho de adolescentes é visto como prejudicial para os jovens de classe média e alta, ele é considerado benéfico para os jovens de classe baixa (Amazarray, Thomé, Souza, Poletto, \& Koller, 2009; Kingston \& Rose, 2015; Leventhal, Graber, \& Brooks-Gunn, 2001; Purtell \& McLoyd, 2013), embora haja estudos que mostrem que um trabalho intenso (aci- 
ma de 20 horas semanais) pode ser prejudicial para os jovens de qualquer classe social (Purtell $\&$ McLoyd, 2013) e que adolescentes pobres em trabalhos dessa natureza tendem a ter comportamentos nocivos em um futuro breve - como o uso de cigarro, álcool e diversidade de parceiros sexuais (Kingston \& Rose, 2015) ou ainda problemas na vida acadêmica, na saúde e na formação da identidade (Amazarray et al., 2009).

Essa inserção antecipada no mundo do trabalho reflete, portanto, uma reprodução social da pobreza, amplamente discutida na literatura nacional e estrangeira como um fenômeno intergeracional (Landstedt, Brydsten, Hammarström, Virtanen, \& Almquist, 2016; Naiff, 2014). Em vários países, o segmento juvenil com problemas socioeconômicos se insere no mercado de trabalho de forma antecipada, visando sua própria sustentação e a de sua família (Pais, 2016). Nos centros urbanos brasileiros, os jovens com maior vulnerabilidade social (negros, baixa escolaridade, oriundos de famílias pobres, sexo feminino) têm mais chances de estarem em situações de trabalho muito precárias e com baixa remuneração (Oliveira, 2009), além de ser uma população que sofre de falta de oportunidade dada ao jovem e de carência de qualificação (Calazans, de Sousa, \& Fischer, 2014).

Estudos no Brasil mostram que é comum o adolescente abandonar o estudo pela necessidade de trabalho, ou pela dificuldade de conciliar trabalho e estudo (Schwartzman \& Castro, 2013). Metade dos brasileiros que concluem o Ensino Médio apresenta distorção de idade-série (IBGE, 2016), o que não está associado somente ao trabalho precoce, mas também à baixa capacidade do sistema educacional de atrair, reter e qualificar os estudantes. Nesse sentido, políticas públicas voltadas para o ingresso de adolescentes no mundo do trabalho precisam levar em conta o incentivo à continuidade dos estudos.

O Programa da Lei da Aprendizagem, conhecido como Programa Jovem Aprendiz - PJA, objeto de estudo do presente artigo, caracteriza-se por promover a inserção laboral de adolescentes e jovens no mundo do trabalho, com a exigência de manutenção do participante na escola. O Programa é vinculado ao Ministério do Trabalho e Emprego - MTE, que estabelece as relações jurídicas, incluindo a prestação de serviços e o contrato de aprendizagem. O PJA considera os dispositivos da Consolidação das Leis Trabalhistas - CLT (Decreto-lei N ${ }^{\circ}$ 5.452, 1943; MTE, 2014) e do Estatuto da Criança e do Adolescente - ECA (Lei No 8069, 1990) para garantir o direito do jovem à profissionalização $\mathrm{e}$ prevê o trabalho com uma formação técnico-profissional compatível com o desenvolvimento físico, moral e psicológico do adolescente (MTE, 2014; Oliveira, 2009).

As características e os objetivos do Programa - voltados para uma inserção laboral apoiada no binômio educação-trabalho - relacionam seus resultados ao desenvolvimento profissional e ao aumento da empregabilidade e da percepção de autoeficácia dos adolescentes e jovens participantes. Assim é que a presente pesquisa se propôs a avaliar a efetividade de tal Programa na vida dos adolescentes. Nessa linha, a questão norteadora definida para a pesquisa foi: Em que medida a participação no PJA contribui para uma percepção mais positiva de autoeficácia, desenvolvimento profissional e empregabilidade por parte dos adolescentes? A pesquisa objetiva, portanto, avaliar a contribuição do Programa Jovem Aprendiz na vida do adolescente de 15 a 18 anos, em termos de desenvolvimento profissional, empregabilidade e autoeficácia. A Tabela 1 apresenta a Síntese do programa Jovem Aprendiz.

Assim, o contrato de aprendizagem vai além de empregar e pagar o salário, pois envolve também aspectos de formação profissional (Oliveira, 2009). Formação profissional faz referência a processos educativos nas escolas ou nas empresas no sentido de absorver e desenvolver conhecimentos, tendo destaque sua natureza processual (Cattani, 2002; Mourão \& Puente-Palacios, 2006). O tema formação profissional, além de ter múltiplos conceitos, está presente nos diferentes momentos da história do Brasil, atrelado ao caráter econômico capitalista (Frigotto, Ciavatta, \& Ramos, 2014) e demandando reflexões referentes aos tradicionais sistemas de educação da sociedade urbano-industrial (Pochmann, 2012). 
Tabela 1

Síntese do Programa Jovem Aprendiz

Dispositivos legais

Agem

Público-alvo

Contrato de

Aprendizagem

Pressuposto básico

Extinção do contrato

Funcionamento

da formação

Tipos de cursos

Instituição formadora

Empresa contratante

Seleção, Remuneração e Jornada

Direitos

Fiscalização

Desenho da Formação
Decreto-lei No 5.452/1943 - CLT, cap. IV, art. 402 à 441; Lei No 10.097/2000; Decreto $\mathrm{N}^{\mathrm{o}} 5.598 / 2005$

Formação técnico-profissional metódica de adolescentes e jovens, desenvolvida por meio de atividades teóricas e práticas, com complexidade progressiva.

Jovens entre 14 a 24 anos, que estejam cursando a escola regular.

Acordo de trabalho especial, por escrito, com prazo máximo de dois anos (exceto jovens com deficiência), em que o empregador assegura formação técnica profissional compatível com desenvolvimento físico, moral e psicológico do aprendiz.

Registro na carteira de Trabalho e Previdência Social - Carteira de Trabalho e Previdência Social [CTPS], assegurados todos os direitos trabalhistas e previdenciários.

Desempenho insuficiente ou inadaptação do aprendiz, falta disciplinar grave, ausência injustificada ou a pedido do aprendiz

O PJA prevê um número máximo de 20 a 30 horas semanais, sendo que 4 a 6 horas dessa carga horária deve ser destinada à formação profissional.

Vários são os cursos de formação profissional que o adolescente pode participar no PJA (ex: ocupações administrativas, práticas bancárias, auxiliar de produção industrial, telemática, telesserviços, turismo e hospitalidade).

Instituições do Sistema S (Serviço Nacional de Aprendizagem Industrial [Senai], Serviço Nacional de Aprendizagem Comercial [Senac], Serviço Nacional de Aprendizagem do Transporte [Senat], Serviço Nacional de Aprendizagem Rural [Senar], Serviço Nacional de Aprendizagem do Cooperativismo [Sescoop]); Escolas técnicas, inclusive agropecuárias; Entidades sem fins lucrativos, registradas nos Conselhos Municipais dos Direitos da Criança e do Adolescente (CMDCAs)

Obrigatoriedade de contratação em estabelecimentos de qualquer natureza, facultado para microempresas (ME) e as entidades sem fins lucrativos; cota de contratação de $5 \%$ a $15 \%$ do total de funcionários; recebe incentivos fiscais ( $2 \%$ de Fundo de Garantia do Tempo de Serviço [FGTS], dispensa aviso prévio e multa rescisória).

Liberdade de seleção pelo empregador, respeitados princípios constitucionais; Salário mínimo/hora, computadas as horas destinadas às atividades teóricas e práticas, nas instituições formadoras e empresas, com limite de até seis horas diárias para jovens cursando o Ensino Fundamental e até oito para jovens no Ensino Médio.

$13^{\circ}$ salário, vale-transporte, férias, FGTS, seguridade social (Instituto Nacional do Seguro Social [INSS]).

Secretaria de Políticas Públicas de Emprego - SPPE e as coordenações de fiscalização de aprendizagem de cada Superintendência Regional do Trabalho e Emprego - SRTE.

Prevê a execução de atividades teóricas e práticas, tendo como objetivo principal a formação profissional do aprendiz. O programa deve considerar o público-alvo (aprendizes por turma e perfil socioeconômico), os conteúdos a serem desenvolvidos em termos de conhecimentos, habilidades e atitudes, bem como a estrutura de programa de aprendizagem conforme o Código Brasileiro de Ocupação (CBO), arco ocupacional, ou em nível técnico médio. 
Algumas pesquisas sobre o PJA são úteis para compreender melhor o programa e seus resultados. O estudo de Pessoa, Alberto, Máximo e Souza (2014) evidencia que os adolescentes percebem uma distância entre o conteúdo da formação profissional e a prática nas empresas, com um processo que privilegia o enquadramento dos jovens no perfil organizacional. Numa linha semelhante, Macêdo e Alberto (2012) confirmam o caráter disciplinador da formação do Jovem Aprendiz, sendo que os egressos dão destaque à comprovação da experiência profissional e à remuneração que permite a aquisição de bens de consumo, com baixa valorização do aprendizado proveniente da participação no programa.

Algo similar ocorre no estudo de Mattos e Chaves (2010), em que os jovens pesquisados, apesar de reconhecerem que desenvolvem novas competências e habilidades, vivenciam os contextos da escola e do trabalho como dois mundos distintos, com tensões e contradições. Mas houve estudos, como o realizado por Amazarray et al. (2009), em que além da valorização da remuneração, os adolescentes também fizeram associação com a aquisição de conhecimento ao longo da vida e valorização da qualificação profissional como meio para conseguir uma boa inserção no mercado.

Na pesquisa de Sousa, Frozzi e Bardagi (2013), a vivência dos adolescentes no PJA resultou em uma avaliação positiva da oportunidade de inserção no mercado de trabalho; e uma avaliação negativa quanto à parte teórica, apesar de reconhecerem que o programa aumenta a motivação para o estudo. Por fim, a pesquisa de Silva e Trindade (2013) identificou que as meninas participantes do PJA valorizam mais a preparação para a vida em termos de crescimento profissional e pessoal, enquanto nos meninos predominam as expectativas de ordem financeira para ajudar a família e obter independência.

Tendo em vista os objetivos estabelecidos para o PJA, sua vinculação com o ECA e as suas características de formação profissional, o presente estudo considera três possíveis variáveis como consequentes do PJA, a saber: desenvolvimento profissional, empregabilidade e autoe- ficácia. Cada uma delas originou, portanto, uma hipótese para a presente pesquisa.

O desenvolvimento profissional refere-se a um processo de aprendizagem relacionado ao trabalho por meio de treinamento ou de educação, considerando o que uma pessoa espera da sua profissão (VandenBos, 2010). O desenvolvimento profissional constitui-se, portanto, num processo de crescimento contínuo nos domínios cognitivo, afetivo e comportamental, que favorece o desempenho no trabalho e o crescimento individual na carreira (Mourão, Porto, \& Puente-Palacios, 2014). Assim, as ações referentes ao desenvolvimento profissional decorrem tanto de aprendizagem formal como informal, propiciando a aquisição de competências (Mourão, Monteiro, \& Viana, 2014). No caso do PJA, o adolescente aprendiz encontra-se numa condição de vivenciar esses dois tipos de aprendizagem, que em seu conceito mais amplo deve ser entendida como um fenômeno existencial e um processo da essência humana, moldada pela interação da pessoa com o mundo, ao longo da vida (Jarvis, 2013). Nesse sentido, acredita-se que o adolescente que participa do PJA perceba uma evolução no seu preparo para a atuação no mundo do trabalho e, por isso, formulou-se a Hipótese 1 - Os adolescentes participantes do PJA percebem-se com maior desenvolvimento profissional do que os adolescentes que não participam do programa.

A empregabilidade, por sua vez, é um tema muito debatido nos meios acadêmicos internacionais e possui uma trajetória desde o início do século XX, com os artigos de Bernard Gazier. Naquela época, o termo abrangia a condição de uma pessoa ser ou não empregável, no sentido de estar imediatamente disponível para o mercado de trabalho, desejando trabalhar e sem impedimentos físicos ou excessivas obrigações familiares (Hirata, 1997). A década de 1990, retoma de forma mais sistemática o conceito de empregabilidade, com maior investigação empírica do seu significado (Forrier \& Sels, 2003), sendo o termo incluído no vocabulário organizacional (Rueda, Martins, \& Campos, 2004). Nesse novo contexto, a empregabilidade passa a ser um conceito 
com espaço nas políticas governamentais, entendido como um construto que interessa à sociedade como um todo e que compõe as políticas de bem-estar social (Adam, Atfield, \& Green, 2017). A empregabilidade como possibilidade de adquirir um trabalho passa a ser entendida, portanto, como consequência dos investimentos de diferentes atores, a saber: os indivíduos com a assunção da responsabilidade pela própria carreira; as organizações com a promoção de possibilidades de desenvolvimento profissional aos seus empregados; e o Estado, com políticas públicas que contribuam para a formação e qualificação profissional (Peixoto, Janissek, \& Aguiar, 2015). Considerando o exposto, acredita-se que a experiência inicial no mundo do trabalho proporcionada pelo PJA, bem como a formação que complementa esta experiência, poderiam gerar nos jovens participantes um aumento na percepção de empregabilidade. Assim foi estabelecida, para a pesquisa, a Hipótese 2 - Após um período de participação no PJA, os adolescentes percebem-se com maior empregabilidade do que aqueles que não participam do programa.

Por fim, o conceito de autoeficácia é apresentado por Albert Bandura, e baseia-se na perspectiva de que a pessoa seria um "agente" de si mesmo, no sentido de influenciar intencionalmente seu próprio funcionamento e suas circunstâncias de vida, no caminho da mudança e do autodesenvolvimento. Nessa concepção, as pessoas são autorreguladas e autorreflexivas, com capacidade de organizar e executar as ações necessárias para atingir e produzir determinado desempenho (Bandura, 2008). As crenças de autoeficácia influenciam, portanto, os comportamentos das pessoas (Pei, Pei, Cheng-Hao, \& Peng-Cheng, 2017), e estão relacionadas às decisões de carreira profissional (Kim, Rhee, Ha, Yang, \& Lee, 2016). Na adolescência, diante das novas responsabilidades assumidas, a autoeficácia está em processo de desenvolvimento e pode atuar como facilitadora nas inúmeras pressões vividas, de forma que a percepção do adolescente sobre tais pressões - vistas como ameaças ou oportunidades - dependa de como ele exerce seu papel de agente (Fontes \& Azzi, 2012). Assim, acredita-se que experiências laborais exitosas advindas do PJA podem aumentar a crença que o adolescente tem de sua própria capacidade. Em função disso, estabeleceu-se a Hipótese 3: Os adolescentes participantes do PJA percebem-se com mais autoeficácia que os adolescentes que não participam do programa.

\section{Método}

$\mathrm{O}$ delineamento desta pesquisa foi de um estudo quase-experimental, com coleta de dados no início e seis meses após o começo da intervenção, considerando-se que esse tempo seria suficiente para os resultados do programa surtirem efeito em termos de desenvolvimento profissional, autoeficácia e percepção de empregabilidade. Havia a intenção de realizar um experimento, que foi inviável por haver uma seleção dos participantes do PJA mediante um perfil desejado pelas organizações contratantes.

Para que o grupo controle mantivesse características semelhantes aos participantes do PJA, foram pesquisados jovens na mesma faixa etária (15 a 18 anos) e que cursavam o Ensino Médio em escolas públicas. Para compor o grupo experimental, foram coletadas informações em quatro instituições formadoras; enquanto o grupo controle foi oriundo de duas escolas públicas. No grupo experimental, a primeira coleta aconteceu no início da formação das turmas e a segunda foi realizada, com os mesmos adolescentes, seis meses após o início da participação nas atividades laborais e formativas do PJA. No grupo controle, foi respeitado o mesmo intervalo de tempo entre a primeira e a segunda coleta.

\section{Participantes}

As condições de inclusão no grupo experimental foram a inscrição e efetiva participação no Programa por pelo menos seis meses. Para os participantes do grupo controle, a condição de inclusão foi ser estudante regularmente matriculado no ensino médio e com idade de 15 a 18 anos. A condição de exclusão para ambos os grupos foi a existência de $30 \%$ ou mais de perguntas em branco nos respectivos questionários 
ou a mortalidade experimental no segundo momento de coleta de dados.

A amostra total foi de 509 jovens, de ambos os sexos, sendo 250 participantes do PJA e 259 adolescentes que compuseram o grupo controle. A distribuição amostral foi de 51,3\% mulheres e a idade variou de 15 anos (10\%) a 18 anos $(27,3 \%)$, com concentração entre 16 e 17 anos $(62,7 \%)$. O maior percentual de pesquisados concentrou-se no primeiro e segundo anos do Ensino Médio (77,2\%) e a escolaridade familiar indicou predomínio de pelo menos um membro da família com Ensino Médio completo (43,5\%) ou incompleto $(20,1 \%)$.

A comparação dos dois grupos (experimental e controle) mostrou dados semelhantes em termos de sexo (Tabela 2). Nas demais variáveis observam-se algumas diferenças entre os grupos. O grupo experimental apresenta uma escolaridade familiar ligeiramente mais elevada que o grupo controle e há também diferença de renda familiar, sobretudo nos estratos mais elevados, pois se comparados os que ganham mais de três salários mínimos, o percentual no grupo experimental é de $21,6 \%$, contra $11,6 \%$ no grupo controle.

A perda amostral entre a primeira e a segunda coleta de dados foi de 107 casos, com menor incidência de desligamento do PJA (32 participantes) quando comparado aos casos de afastamento escolar (75 no grupo controle). Esse resultado pode ser explicado pelo fato de a coleta de dados do segundo momento ter sido realizada no final do ano letivo, período em que muitos alunos já sabem que não serão aprovados e param de frequentar a escola. Nas análises foram, portanto, considerados somente os adolescentes que participaram das duas coletas, sendo 218 do grupo experimental e 184 do grupo controle, cuja perda foi mais acentuada.

\section{Instrumentos de Coleta de Dados}

Na presente pesquisa, optou-se por trabalhar com instrumentos que já tivessem evidências de validade psicométrica. Assim, a percepção do desenvolvimento profissional foi mensurada a partir da Escala de Percepção Atual do Desenvolvimento Profissional - EPADP, desenvolvi- da por Mourão, Porto, et al. (2014), unifatorial, composta de oito itens, variando de 0 (discordo totalmente) a 10 (concordo totalmente) e grau de precisão de 0,82 (Alpha de Cronbach). Os itens da escala foram ligeiramente adaptados para se adequar à realidade do PJA, por exemplo: "Tive um expressivo desenvolvimento profissional desde que comecei a trabalhar" foi modificado para "Tive um expressivo desenvolvimento profissional desde que comecei no PJA".

Para a autoeficácia, foi utilizada uma versão reduzida da Escala Autoeficácia Geral Percebida - EAGP, de Nunes, Schwarzer e Jerusalém (1999), adaptada para amostras brasileiras por Martins e Onça (2011). A escala é unifatorial, composta por 5 itens, que variaram de 0 (discordo totalmente) a 10 (concordo totalmente). Ex. de item: "Eu acredito que posso realizar de forma eficaz muitas tarefas diferentes". O índice de consistência (Alpha de Cronbach) obtido no estudo inicial foi de 0,81 .

Por fim, também foi utilizada a Escala de Autopercepção de Empregabilidade - EAE, desenvolvida por Peixoto et al. (2015), que tem por objetivo avaliar a percepção dos indivíduos em relação ao seu grau de empregabilidade. Ela é composta por dois fatores - Manutenção (do emprego atual) e Aquisição (de um novo emprego) - com 10 itens, respondidos com base em uma escala do tipo Likert de seis pontos, variando entre 0 (discordo totalmente) e 10 (concordo totalmente). Tendo em vista que a Dimensão Manutenção diz respeito a pessoas que já têm um emprego - o que não condiz com os jovens aprendizes -, nesta pesquisa foi utilizada somente a dimensão Aquisição, com quatro itens e índice de consistência (Alpha de Cronbach) de 0,75. Ex. de item: "Acredito que outra empresa tem interesse em me contratar".

\section{Procedimentos Éticos}

A pesquisa foi inscrita na Plataforma Brasil e previamente aprovada por um comitê de ética (CAEE 47586215.7.0000.5289). Os participantes maiores de 18 anos e os responsáveis pelos participantes menores de 18 anos assinaram o Termo de Consentimento Livre e Esclarecido e 


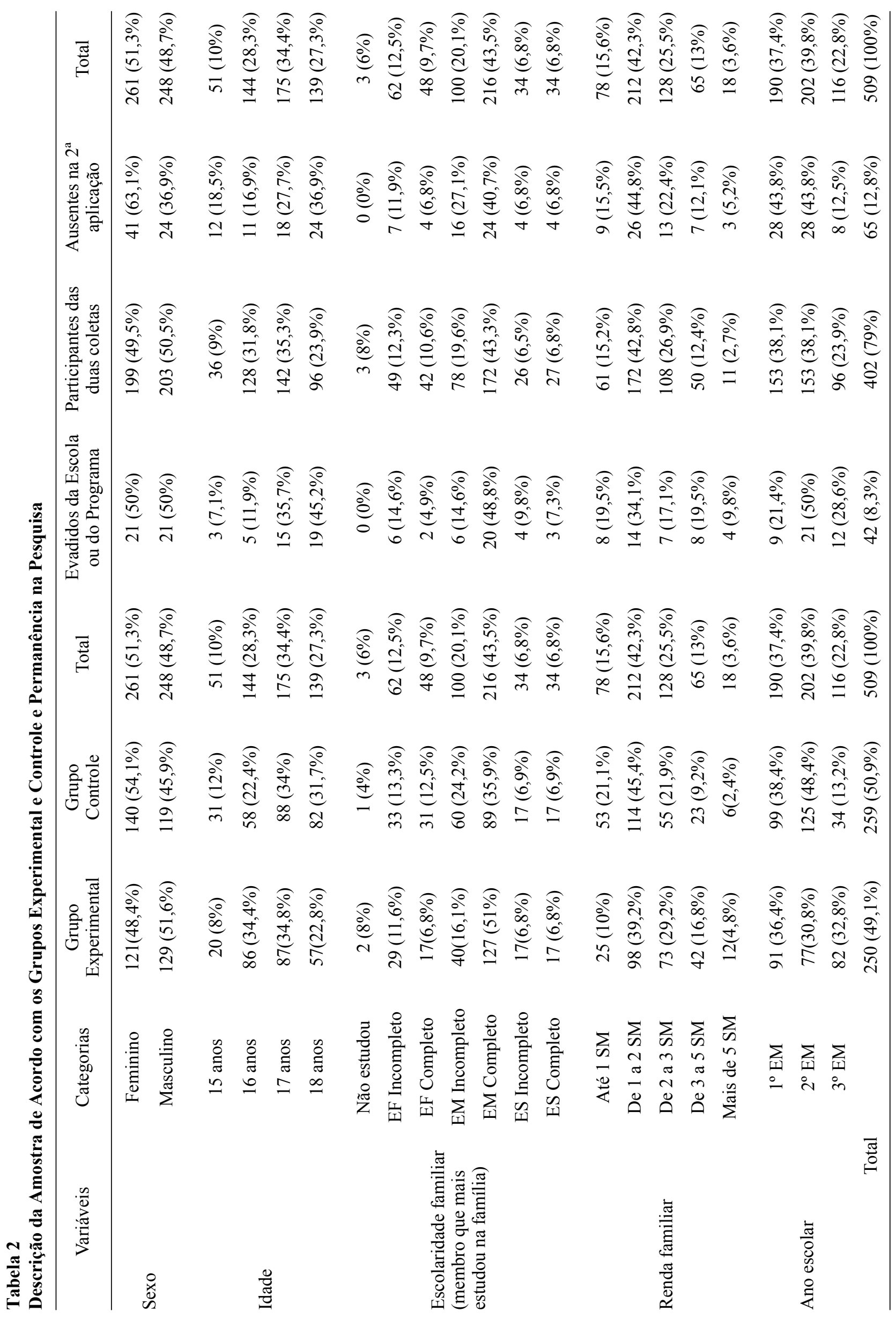


foi garantido aos participantes o direito a conhecer os objetivos e os resultados da pesquisa, tendo sido disponibilizado um retorno individualizado dos resultados para todos os adolescentes que se interessaram. Foi preservado o sigilo das informações individuais obtidas ao longo da coleta de dados. A aplicação dos questionários foi feita pela própria pesquisadora, sem a interveniência de outras pessoas no processo de coleta de dados.

\section{Procedimentos de Coleta de Dados}

O tempo médio de cada aplicação foi de 40 minutos. As instituições participantes tiveram acesso ao projeto de pesquisa e ao resultado de aprovação do Comitê de Ética, havendo concordância explícita dos representantes dessas instituições em colaborar com a pesquisa.

Conforme previsto no delineamento, a pesquisa contemplou dois grupos de adolescentes: participantes do PJA (grupo experimental) e não participantes (grupo controle). Para ambos os grupos, a coleta de dados ocorreu em dois momentos distintos, com intervalo de seis meses entre eles. O primeiro momento de coleta foi definido como a semana inicial de participação no PJA, respeitando-se o mesmo calendário de aplicações para os alunos da rede pública que não participam do programa (grupo controle).

\section{Procedimentos de Análise de Dados}

A tabulação e a análise dos dados quantitativos foram realizadas com o auxílio do Software Statistical Package for Social Science - SPSS, versão 21.0. Para verificar a existência de diferenças entre as medidas de desenvolvimento profissional, empregabilidade e autoeficácia utilizadas no pré e pós-teste, foi realizada uma ANOVA mista 2 (grupo) X 2 (tempo). Antes de analisar a ANOVA propriamente dita, foi feito o exame dos pressupostos para esse tipo de teste. Nesse sentido, verificou-se inicialmente que as variáveis dependentes (desenvolvimento profissional, empregabilidade e autoeficácia) eram normalmente distribuídas, que as variâncias dos grupos eram homogêneas e que não havia a presença de outliers (escores extremos) em nenhuma das distribuições, o que evidenciou a perti- nência da ANOVA ser adotada na comparação entre as médias dos grupos experimental e de controle.

\section{Resultados}

No que diz respeito à percepção de desenvolvimento profissional, a ANOVA indicou que a interação entre grupo e tempo foi significativa, com escores dos participantes do PJA maiores após seis meses no Programa, quando comparados escores do grupo controle decorrido o mesmo intervalo temporal (Tabela 3), sendo, portanto, verificada diferença significativa entre o pré e o pós-teste nos grupos experimental e controle, $F(1)=42,07 ; p<0,01$. Apesar de ser observada uma diferença inicial entre os escores de desenvolvimento profissional dos grupos experimental e controle, os resultados indicaram uma significativa acentuação dessas diferenças após seis meses de participação ou não no Programa (Figura 1), ficando o grupo controle com escores estáveis, enquanto o grupo experimental apresentou elevação dos escores de desenvolvimento profissional.

No que diz respeito à autopercepção de empregabilidade, a interação entre grupo e tempo foi significativa, sendo os escores de percepção de empregabilidade dos participantes do PJA maiores após seis meses no Programa, quando comparados aos escores do grupo controle no mesmo período, $F(1)=19,35 ; p<0,01$. Os dados mostram escores estatisticamente equivalentes no pré-teste (Tabela 3), mas decorridos os seis meses da aplicação inicial, o grupo experimental passa a apresentar escores mais elevados de percepção de empregabilidade do que o grupo controle, indicando que os seis meses de participação no PJA favoreceram a melhoria de percepção de empregabilidade no adolescente (Figura 2).

No que diz respeito à autoeficácia, a interação entre grupo e tempo foi significativa, confirmando que os escores de autoeficácia dos participantes do PJA foram maiores após seis meses no Programa, quando comparados aos valores obtidos pelo grupo controle. O resultado da ANOVA comparando a diferença entre o 
Tabela 3

Resultados da ANOVA para as Variáveis Desenvolvimento Profissional, Autoeficácia e Empregabilidade

\begin{tabular}{lccccc}
\hline \hline $\begin{array}{l}\text { Variáveis/ } \\
\text { Fonte de variância }\end{array}$ & $\begin{array}{c}\text { Soma dos } \\
\text { quadrados }\end{array}$ & $G L$ & $\begin{array}{l}\text { Quadrados da } \\
\text { média }\end{array}$ & $F$ & $p$ \\
\hline Desenvolvimento Profissional & & & & & \\
$\quad$ Grupo & 157,96 & 1 & 157,96 & 67,68 & 0,01 \\
Erro (Grupo) & 926,53 & 397 & 2,33 & & \\
Tempo & 64,23 & 1 & 64,23 & 45,86 & 0,01 \\
Erro (Tempo) & 556,11 & 397 & 1,40 & & \\
Grupo X Tempo & 58,93 & 1 & 58,93 & 42,07 & 0,01 \\
Autoeficácia & & & & & \\
Grupo & 0,27 & 1 & 0,27 & 0,18 & 0,67 \\
Erro Grupo & 586,71 & 396 & 1,48 & & \\
Tempo & 17,95 & 1 & 17,95 & 15,98 & 0,01 \\
Erro (Tempo) & 444,70 & 396 & 1,12 & & \\
Grupo X Tempo & 12,30 & 1 & 12,30 & 10,95 & 0,01 \\
Empregabilidade & & & & & \\
Grupo & 1,13 & 1 & 1,13 & 0,76 & 0,38 \\
Erro (Grupo) & 591,95 & 399 & 1,48 & & 5,86 \\
Tempo & 6,90 & 1 & 6,90 & 0,02 & \\
Erro (Tempo) & 469,61 & 399 & 1,18 & & \\
Grupo X Tempo & 22,77 & 1 & 22,77 & 19,35 & 0,01 \\
\hline
\end{tabular}

pré e o pós-teste nos dois grupos (experimental e controle) foi, portanto, significativo, $F(1)$ $=10,95 ; p<0,01$. Vale ressaltar, que da mesma forma que ocorreu com a empregabilidade, também em relação à autoeficácia não houve diferença significativa nos escores iniciais dos dois grupos (Tabela 3). Portanto, os seis meses de participação no PJA favoreceram a melhoria de autoeficácia no adolescente (Figura 3).

\section{Discussão}

O presente estudo avaliou o PJA a partir da comparação das percepções de desenvolvimento profissional, empregabilidade e autoeficácia de um grupo de adolescentes participantes do programa e um grupo controle de alunos do Ensino Médio que não participaram do PJA. A
Hipótese 1 ("Os adolescentes participantes do PJA se percebem com maior desenvolvimento profissional do que os adolescentes não participantes") foi confirmada, pois transcorridos seis meses de participação no Programa, os adolescentes se perceberam com mais crescimento profissional. Considerando a amplitude e a complexidade do conceito de formação profissional (Cattani, 2002; Mourão \& Puente-Palacios, 2006), o resultado aponta para a ocorrência de um processo educativo que está surtindo efeitos possivelmente em função da experiência nas empresas e no curso que ocorre nas instituições formadoras. Ao que tudo indica, a combinação Escola-Trabalho tem permitido que os adolescentes absorvam conhecimentos teórico, técnico e operacional voltados para a produção de bens e serviços, de forma a avaliarem mais positiva- 


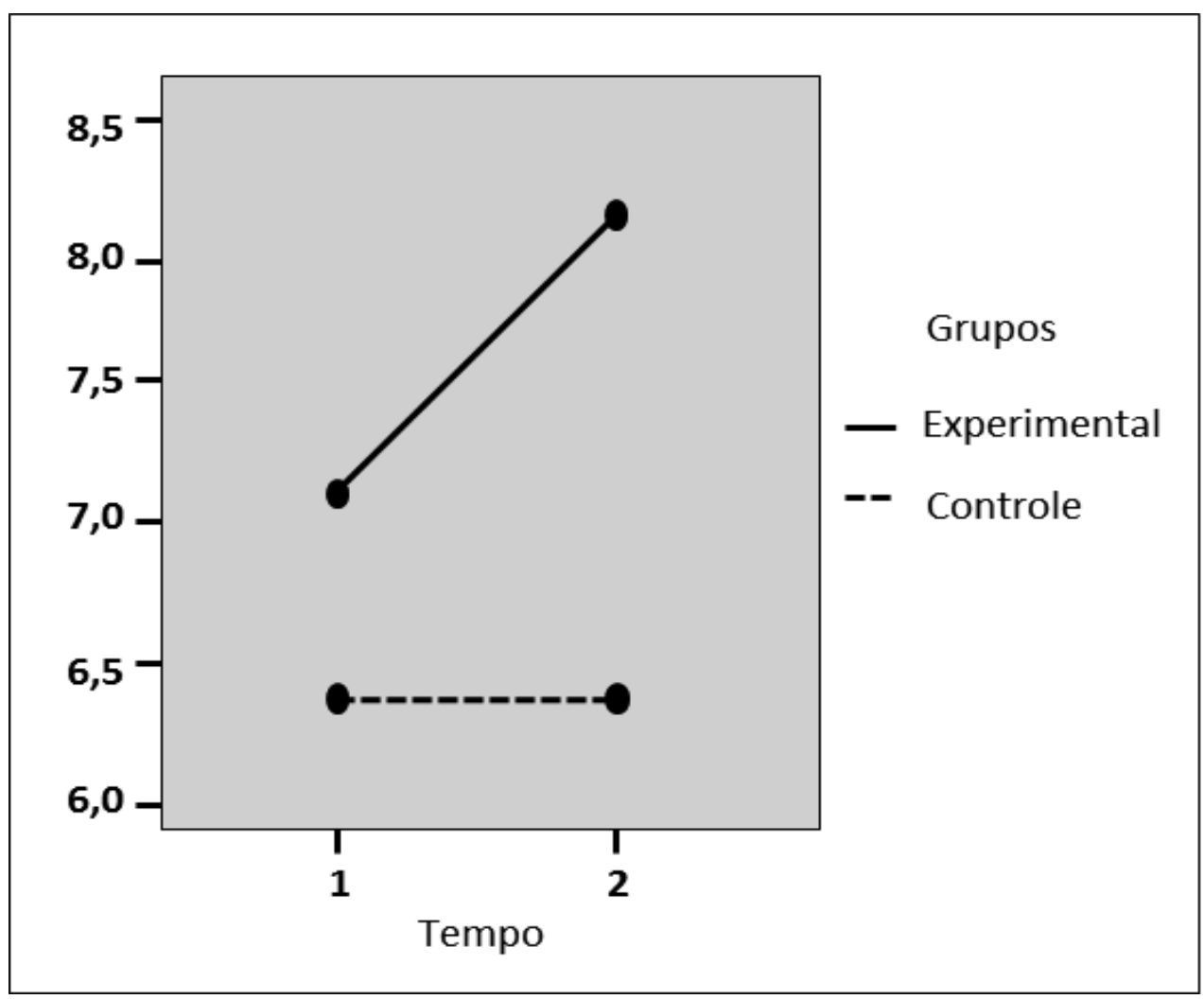

Figura 1. Interação entre grupo e tempo em termos de desenvolvimento profissional.

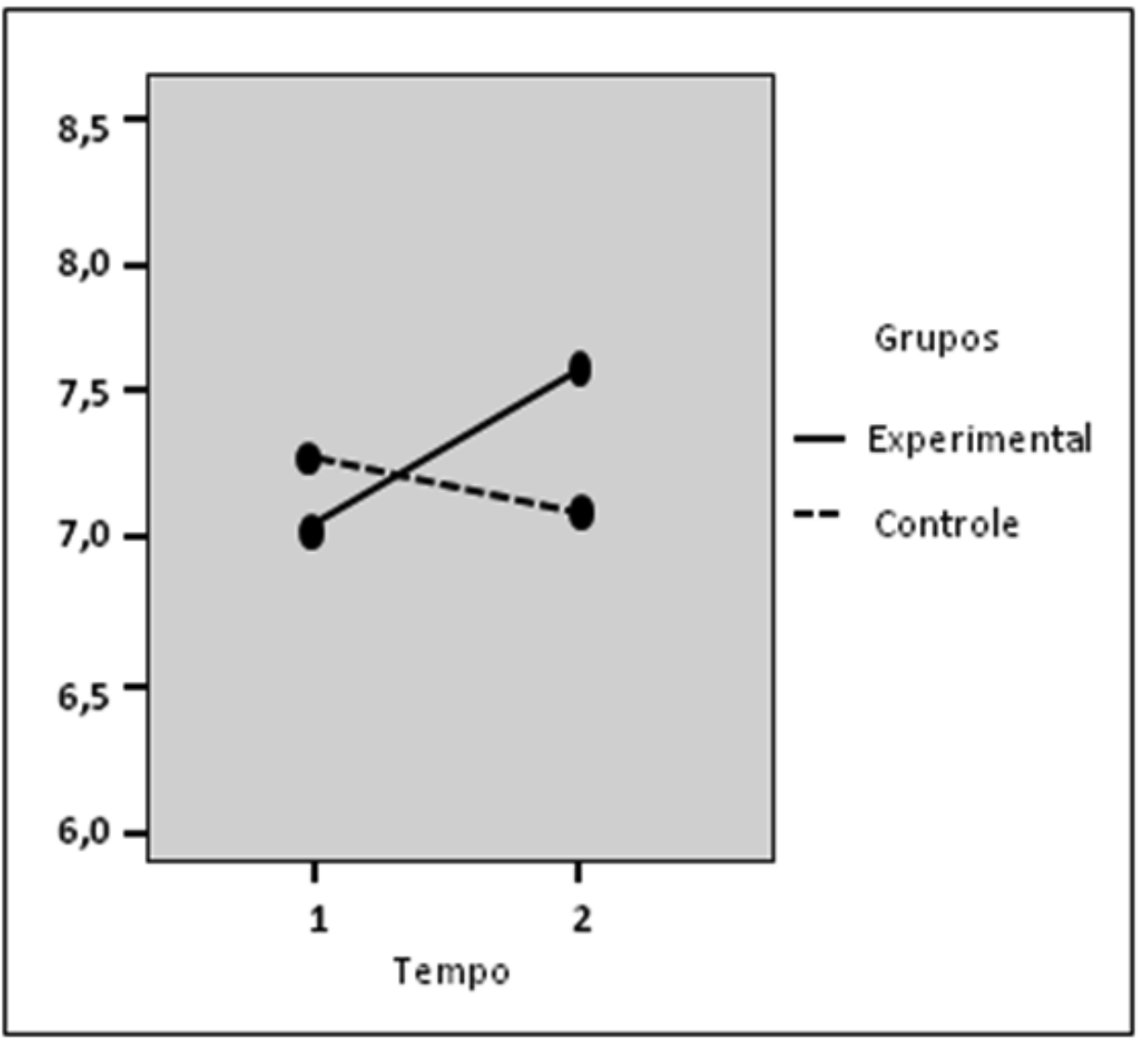

Figura 2. Interação entre grupo e tempo em termos de empregabilidade. 


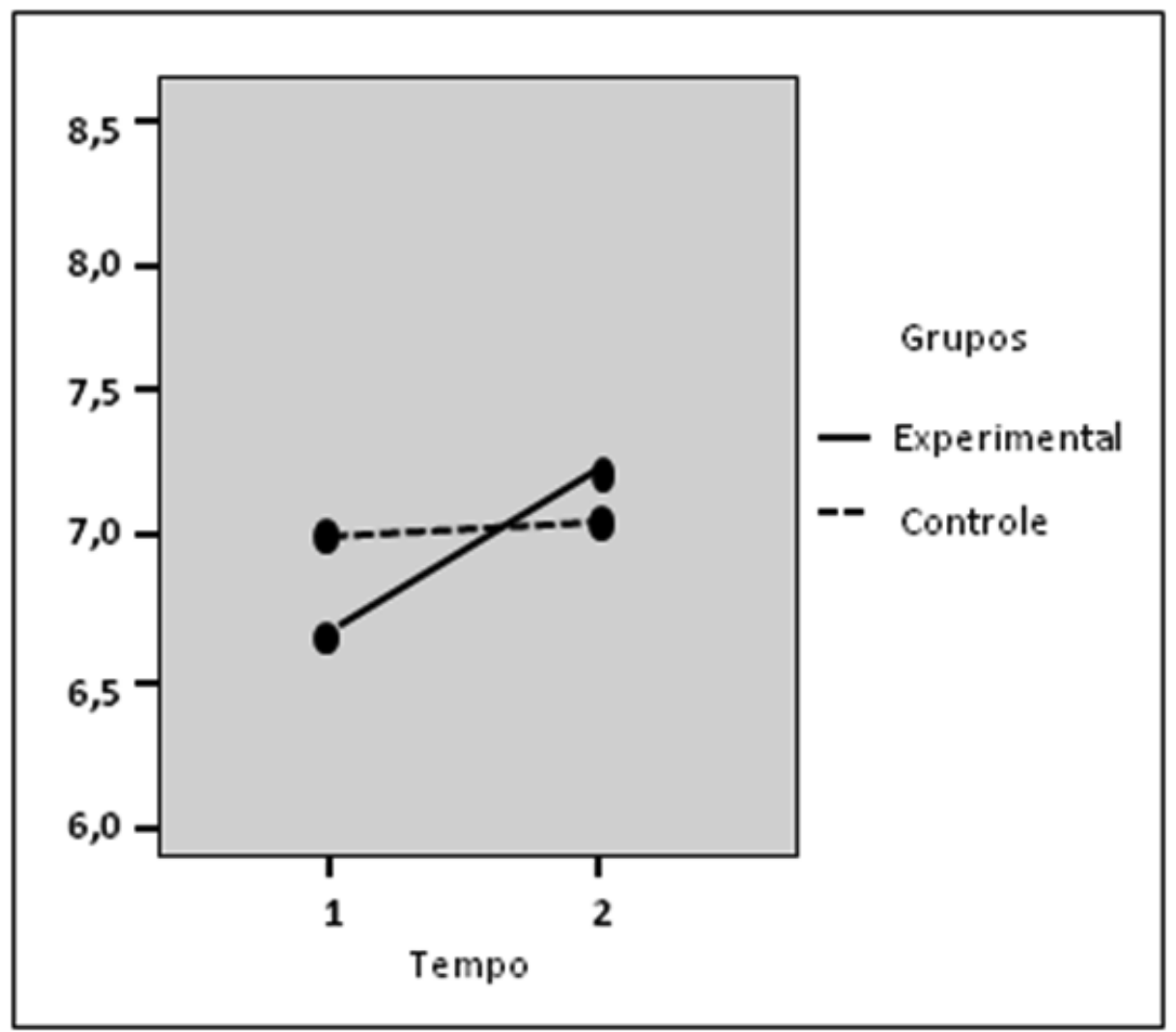

Figura 3. Interação entre grupo e tempo em termos de autoeficácia.

mente o seu desenvolvimento profissional. Tal desenvolvimento também pode estar associado a uma permanência na escola, uma vez que a presente pesquisa indicou maior evasão escolar entre os participantes do grupo controle. Esse resultado pode estar associado à exigência do PJA de que o adolescente se mantenha na escola para poder continuar participando do programa (MTE, 2014), além de confirmar estudos anteriores que já apontavam menor evasão escolar entre adolescentes que trabalhavam (Leventhal et al., 2001).

Esse resultado também permite inferir que a participação em processos de aprendizagens formais e informais favorece a aquisição de competências e se constitui em ações importantes para o desenvolvimento profissional (Mourão, Monteiro, et al., 2014), pois o adolescente participante do programa está em processo de aprendizagem tanto nas instituições formadoras como nas atividades laborais. Acredita-se, portanto, que a articulação entre o ambiente educacional e o empresarial desenvolva uma aprendizagem teórica e prática, favorecendo o processo educativo e a realização pessoal dos participantes (Frigotto et al., 2014).

O fato de o grupo experimental ter iniciado a pesquisa com escores de desenvolvimento profissional ligeiramente inferiores aos escores do grupo controle e, após seis meses, esse resultado ter se invertido, com uma diferença significativa entre os grupos pode confirmar a capacidade de transformação do processo educativo (Jarvis, 2013) associado ao PJA. Além disso, a presente pesquisa confirma os achados de Mattos e Chaves (2010) que identificaram que, apesar dos adolescentes egressos do PJA perceberem uma distância entre a teoria e a prática, eles reconhecem que o Programa proporciona o desenvolvimento de novas competências e habilidades. $\mathrm{Na}$ pesquisa atual, mesmo considerando que a coleta de dados ocorreu com apenas seis meses do início da participação no Programa, os resultados em termos de desenvolvimento profissional são visíveis.

A segunda hipótese que considera que os adolescentes participantes do PJA obtêm maior percepção de empregabilidade do que os não par- 
ticipantes foi confirmada. Após os primeiros seis meses de formação, os participantes do Programa apresentaram um crescimento significativo em tal percepção. Esse resultado encontra respaldo em conceitos da área que vinculam a percepção de empregabilidade à aquisição de competências individuais e habilidades para realizar tarefas e funções (Peixoto et al., 2015). Como o adolescente inserido no Programa tem oportunidades de ter experiências no mercado de trabalho e de contar com uma formação profissional, há um ganho provável de competências (Mourão, Porto, et al., 2014) que contribui para a percepção de ter mais condições de ser empregado.

Considerando que os participantes se encontram na fase de adolescência, de transformação, autoafirmação e busca pela autonomia (Cerqueira-Santos et al., 2014), a vivência profissional contribui para concretizar esse movimento de independência (Silva \& Trindade, 2013). Assim, ser selecionado para participar do PJA e ter sua primeira experiência no mundo do trabalho ajuda os adolescentes a se perceberem como seres mais independentes e autônomos no seu processo de desenvolvimento e com mais chances de inserção definitiva no mercado de trabalho (Sousa et al., 2013).

Em outra perspectiva, considerando que a empregabilidade não se caracteriza exclusivamente como variável individual (Adam et al., 2017; Peixoto et al., 2015; Rueda et al., 2004), é preciso refletir acerca do risco de a participação no PJA criar ilusões de que será fácil conseguir um emprego após o término do programa. O momento econômico do país e outras variáveis de nível macro podem dificultar que esses jovens consigam um emprego após o término do contrato do Programa, sobretudo considerando que os jovens são os que mais sofrem com o aumento do desemprego no país (IBGE, 2016).

$\mathrm{Na}$ confirmação da hipótese sobre empregabilidade, cabem outras discussões. Se, por um lado, os participantes do PJA percebem um crescimento da sua empregabilidade; por outro, os adolescentes que cursam o Ensino Médio em escolas públicas e não participam do Programa apresentam resultado oposto. O passar do tempo faz com que eles se conside- rem menos empregáveis. Tal resultado possivelmente confirma a análise de Calazans et al. (2014) de que grande parte da população jovem, principalmente a de maior vulnerabilidade social, busca se inserir no mercado de trabalho e se depara com a indisponibilidade de oportunidade e com a carência de qualificação. Assim, tentativas frustradas de conseguir um emprego podem contribuir para que esses adolescentes tenham uma queda na sua percepção de empregabilidade na medida em que passa o tempo. Além disso, o resultado também aponta para um fracasso da educação básica no sentido de preparação para o trabalho, mesmo entre os jovens de classes sociais mais baixas, para os quais haveria uma expectativa de que o Ensino Médio contribuísse para a formação profissional (Frigotto et al., 2014).

Com relação à terceira hipótese da pesquisa, os adolescentes participantes do PJA desenvolveram uma melhor percepção de autoeficácia do que os não participantes, confirmando a hipótese. Esse resultado, provavelmente, relaciona-se à teoria de Bandura (1977) de que as experiências de êxito são fundamentais para o desenvolvimento do senso de autoeficácia. Considerando que a autoeficácia se refere às crenças na própria capacidade de produzir determinados níveis de desempenho (Bandura, 1977), quanto mais elevado o nível de autoeficácia, maior a tendência de escolher atividades desafiadoras (Kim et al., 2016). Nesse sentido, esse é um processo que se retroalimenta e, como na adolescência a autoeficácia está em processo de desenvolvimento, isso pode atuar como facilitador para o início da vida laboral. Assim, o ganho em termos de autoeficácia aponta para uma avaliação pessoal mais positiva acerca da própria capacidade de realização, agindo sobre o estereótipo de ativação (Pei et al., 2017).

Em síntese, as principais conclusões desta pesquisa são: (a) o PJA ao fazer um processo seletivo, acaba por absorver os que se percebem como mais desenvolvidos profissionalmente, mantendo a desigualdade de ofertas na sociedade; (b) o PJA possibilita que o adolescente se perceba com um crescente desenvolvimento profissional, o que não ocorre com aqueles que 
estão no Ensino Médio e não participam do Programa; (c) o adolescente ao se sentir adquirindo um aprendizado profissional por meio do PJA acaba por desenvolver uma melhor percepção de sua empregabilidade, ao contrário do que ocorre com aqueles que estão nas escolas públicas e fora do Programa; (d) as experiências dos adolescentes no PJA contribuem para promover o desenvolvimento de crenças em sua capacidade de atingir resultados favoráveis para a sua vida, ao passo que o jovem do Ensino Médio das escolas públicas vai, ao longo de um tempo, diminuindo a sua percepção de autoeficácia.

Com base nessas conclusões pode-se afirmar que o PJA contribui, positivamente, para que o adolescente se perceba, num curto período de tempo, em condições mais favoráveis do que quando ingressou no Programa em relação às variáveis desenvolvimento profissional, empregabilidade e autoeficácia. Dessa forma, é possível apontar algumas contribuições teóricas do estudo, devendo ser mencionadas: (a) a identificação de uma relação entre experiência laboral e formação profissional na adolescência e a percepção de desenvolvimento profissional; (b) a confirmação de que experiências laborais e de aprendizagem podem contribuir para o desenvolvimento da autoeficácia dos adolescentes; e (c) os indícios de que os jovens conseguem avaliar a sua empregabilidade e que percebem diferenças na mesma, mediante a participação em um curso de formação que envolve o contato com o mundo organizacional.

Além das contribuições teóricas, há também implicações práticas da presente pesquisa, com recomendações tanto para os gestores do PJA, quanto para os educadores que atuam na gestão do Ensino Médio. Para estes, recomenda-se que possam desenvolver soluções que permitam cumprir o que é estabelecido na Constituição Federal e na LDB em termos de preparo dos estudantes para o mundo do trabalho. Essa recomendação ganha relevância quando observamos que a maior parte dos trabalhadores brasileiros tem nível médio de escolaridade. Para essas pessoas, a Educação Básica é a última formação recebida, o que aumenta a responsabilidade do preparo que é ofertado nessa etapa educacional.
Apesar das conclusões alcançadas e do atingimento do objetivo de avaliar a contribuição do Programa Jovem Aprendiz na vida do adolescente de 15 a 18 anos, em termos de desenvolvimento profissional, empregabilidade e autoeficácia, esta pesquisa apresenta algumas limitações. A coleta de dados foi feita num intervalo de 6 meses, que é um tempo inferior à duração do programa (18 a 24 meses). Outra limitação é a delimitação geográfica restrita ao estado do Rio de Janeiro, pois é possível que os resultados do PJA em outras localidades sejam distintos dos que foram obtidos no presente estudo.

A despeito dessas limitações, a pesquisa apresenta contribuições. Mesmo sem ter avaliado o programa em toda a sua extensão, o fato de ter realizado uma pesquisa quase-experimental (com grupo controle e dois momentos de coleta de dados) permitiu identificar que as experiências do PJA trazem resultados positivos para os adolescentes, ainda que seja identificada alguma desigualdade no processo seletivo do Programa.

Para dar continuidade aos estudos da área, seria importante que pesquisas futuras investigassem o adolescente no início e no término do programa, respeitando o período máximo da formação. Além disso, o desenvolvimento de pesquisas qualitativas contribuiria para avaliar o desenvolvimento pessoal do adolescente, com a identificação de sentidos e significados atribuídos pelos mesmos a partir dessa experiência precoce no mundo do trabalho. Por fim, também seriam recomendados estudos mais amplos, com amostras nacionais que permitam identificar os resultados do PJA em outros estados brasileiros.

\section{Referências}

Adam, D., Atfield, G., \& Green, A. E. (2017). What works? Policies for employability in cities. Urban Studies, 54(5), 1162-1177. doi: $10.1177 / 0042098015625021$

Amazarray, M. R., Thomé, L. D., Souza, A. P. L. D., Poletto, M., \& Koller, S. H. (2009). Aprendiz versus trabalhador: Adolescentes em processo de aprendizagem. Psicologia: Teoria e Pesquisa, 25(3), 329-338. doi: 10.1590/S010237722009000300006 
Bandura, A. (1977). Self-efficacy: Toward a unifying theory of behavior change. Psychological Review, 84(2), 191-215.

Bandura, A. (2008). A evolução da teoria social cognitiva. In A. Bandura, R. G. Azzi, \& S. Polydoro, Teoria social cognitiva: Conceitos básicos (pp. 15-41). Porto Alegre, RS: Artmed.

Cattani, A. D. (Ed.). (2002). Trabalho e Tecnologia: Dicionário Crítico. Verbetes: Formação Profissional e Teoria do Capital Humana (4. ed.). Petrópolis, RJ: Vozes.

Calazans, F. D. D., de Sousa, J. P. Z. M., \& Fischer, L. (2014). Programa de aprendizagem profissional e sua contribuição mercadológica: Uma análise sob as perspectivas aprendiz, professor e empresa. Bioenergia em Revista: Diálogos, 4(1), 35-58.

Cerqueira-Santos, E., Melo, O. C., Neto, \& Koller, S. H. (2014). Adolescentes e Adolescências. In L. F. Habigzang, E. Diniz, \& S. H. Koller, Trabalhando com adolescentes: Teoria e intervenção psicológica (pp. 17-29). Porto Alegre, RS: Artmed

Decreto-lei $N^{\circ}$ 5.452, de 01 de maio de 1943. (1943). Aprova a Consolidação das Leis do Trabalho. Diário Oficial da União. Recuperado em http://www2.camara.leg.br/legin/fed/declei/1940-1949/decreto-lei-5452-1-maio-1943-415500-publicacaooriginal-1-pe.html

Decreto $\mathrm{N}^{\mathrm{o}} 5.598$, de $1^{\circ}$ de dezembro de 2005. (2005, 2 dez.). Regulamenta a contratação de aprendizes e dá outras providências. Diário Oficial da União. Recuperado em http://www.planalto. gov.br/ccivil_03/_Ato2004-2006/2005/Decreto/ D5598.htm

Fontes, A. P., \& Azzi, R. G. (2012). Crenças de autoeficácia e resiliência: Apontamentos da literatura sociocognitiva. Estudos Psicologia (Campinas), 29(1), 105-114.

Forrier, A., \& Sels, L. (2003). The concept employability: A complex mosaic. International Journal of Human Resources Development and Management, 3(2), 102-124.

Frigotto, G., Ciavatta, M., \& Ramos, M. (2014). A educação de trabalhadores no Brasil contemporâneo: Um direito que não se completa. Germinal: Marxismo e Educação em Debate, 6(2), 65-76.

Hirata, H. (1997). Os Mundos do Trabalho: Convergência e diversidade num contexto de mudança dos paradigmas produtivos. In A. Casali, I. Rios, J. E. Teixeira, \& M. S. Cortella (Eds.), Emprega- bilidade e educação: Novos caminhos no mundo do trabalho (pp. 23-42). São Paulo, SP: Educ.

Instituto Brasileiro de Geografia e Estatística. (2013). Pesquisa Nacional por Amostra de Domicílios. Sintese de indicadores (PNAD). Rio de Janeiro, RJ: Autor.

Instituto Brasileiro de Geografia e Estatística. (2016). Pesquisa Nacional por Amostra de Domicílios. Sintese de indicadores (PNAD). Rio de Janeiro, RJ: Autor.

Jarvis, P. (2013). Aprendendo a ser uma pessoa na sociedade. In K. Illeris (Ed.), Teorias contemporâneas da aprendizagem (pp. 31-45). Porto Alegre, RS: Penso

Kim, B., Rhee, E., Ha, G., Yang, J., \& Lee, S. M. (2016). Tolerance of uncertainty: Links to happenstance, career decision self-efficacy, and career satisfaction. Career Development Quarterly, 64(2), 140-152. doi: 10.1002/cdq.12047

Kingston, S., \& Rose, A. (2015). Do the effects of adolescent employment differ by employment intensity and neighborhood context?. American Journal of Community Psychology, 55(1), 3747. doi: 10.1007/s10464-014-9690-y

Landstedt, E., Brydsten, A., Hammarström, A., Virtanen, P., \& Almquist, Y. B. (2016). The role of social position and depressive symptoms in adolescence for life-course trajectories of education and work: A cohort study. BMC Public Health, 16(1), 1-16. doi: 10.1186/s12889-016-3820-4

Lei $N^{\circ} 8069$, de 13 de julho de 1990. (1990). Dispõe sobre o Estatuto da Criança e do Adolescente e dá outras providências. Diário Oficial da República Federativa do Brasil. Recuperado em http://www.planalto.gov.br/ccivil_03/Leis/ L8069Compilado.htm

Lei $\mathrm{N}^{\circ}$ 10.097, de 19 de dezembro de 2000. (2000). Altera dispositivos da Consolidação das Leis do Trabalho - CLT, aprovada pelo Decreto-Lei n. 5.452 , de $1^{\circ}$ de maio de 1943. Diário Oficial da União, Seção 1, p. 1. Recuperado em http://www2.camara.leg.br/legin/fed/lei/2000/ lei-10097-19-dezembro-2000-365495-publicacaooriginal-1-pl.html

Leventhal, T., Graber, J. A., \& Brooks-Gunn, J. (2001). Adolescent transitions to young adulthood: Antecedents, correlates, and consequences of adolescent employment. Journal of Research on Adolescence, 11(3), 297-323.

Macêdo, O. J. V., \& Alberto, M. D. F. P. (2012). O sentido da formação profissional no contexto da 
aprendizagem. Estudos de Psicologia (Natal), $17(2), 223-231$.

Martins, M. C. F., \& Onça, S. S. (2011). Escala de autoeficácia percebida: Adaptação e validação. In Anais do III Congresso Ibero-Americano de Psicologia das Organizações e do Trabalho. Florianópolis, SC: Universidade Federal de Santa Catarina.

Mattos, E., \& Chaves, A. M. (2010). Trabalho e escola: É possível conciliar? A perspectiva de jovens aprendizes baianos. Psicologia Ciência e Profissão, 30(3), 540-555.

Ministério do Trabalho e Emprego. (2014). Manual de Aprendizagem: O que é preciso saber para contratar o aprendiz (Ed. revista e ampliada). Recuperado em acesso.mte.gov.br/políticas_juventude/manualdeaprendizagem

Mourão, L., Monteiro, A. C. F., \& Viana, V. R. (2014). A influência do desenvolvimento profissional e da identificação organizacional na satisfação no trabalho. Psico, 45(2), 198-208.

Mourão, L., Porto, J. B., \& Puente-Palacios, K. (2014). Construção e evidências de validade de duas escalas de percepção de desenvolvimento profissional. Psico USF, 19(1), 73-85.

Mourão, L., \& Puente-Palacios, K. E. (2006). Formação profissional. In J. E. Borges-Andrade, G. S. Abbad, \& L. Mourão, Treinamento, desenvolvimento e educação em organizações e trabalho: Fundamentos para a gestão de pessoas (pp. 4164). Porto Alegre, RS: Artmed.

Naiff, L. A. M. (2014). De mãe para filha o legado da exclusão social: Um estudo de memórias autobiográficas. Rio de Janeiro, RJ: Seropédica.

Nunes, R., Schwarzer, R., \& Jerusalem, M. (1999). Auto-eficácia geral percepcionada. Recuperado em http://userpage.fu-berlin.de/gesund/skalen/ Language_Selection/Portuguese/Auto-Eficacia Geral_Percepcion/auto-eficacia_geral_percepcion.htm

Oliveira, O. (2009). Trabalho e profissionalização de adolescente. São Paulo, SP: Editora LTr.

Pais, J. M. (2016). Ganchos, tachos e biscates: Jovens, trabalho efuturo (3. ed.). Porto, Portugal: Ambar.
Pei, W., Pei, Z., Cheng-hao, T., \& Peng-Cheng, Z. (2017). Effect of self-efficacy in stereotype activation. Social Behavior \& Personality: an International Journal, 45(3), 469-476. doi: 10.2224/ sbp.5201

Peixoto, A. D. L. A., Janissek, J., \& Aguiar, C. V. N. (2015). Autopercepção de Empregabilidade In K. Puente-Palacios \& A. D. L. A. Peixoto, Ferramentas de Diagnóstico para Organizações e Trabalho: Um Olhar a partir da Psicologia (pp. 175-186). Porto Alegre, RS: Artmed.

Pessoa, M. C. B., Alberto, M. D. F. P., Máximo, T. A. C. D. O., \& Souza, P. C. Z. D. (2014). Formação profissional de jovens: A que se destina? Estudos de Psicologia (Natal), 19(1), 22-30.

Pochmann, M. (2012). Trabalho e formação. Educação \& Realidade, 37(2), 491-508. Recuperado em http://www.ufrgs.br/edu_realidade

Purtell, K. M., \& McLoyd, V. C. (2013). A longitudinal investigation of employment among low-income youth: Patterns, predictors, and correlates. Youth and Society, 45(2), 243-264.

Rueda, F. J. M., Martins, L. J., \& Campos, K. C. L. (2004). Empregabilidade: O que os alunos universitários entendem sobre isto? Revista Psicologia-Teoria e Prática, 6(2), 63-73.

Schwartzman, S., \& Castro, C. M. (2013). Ensino, formação profissional e a questão da mão de obra. Ensaio: Avaliação de Políticas Públicas em Educação, 21(80), 563-624.

Silva, R. D. M., \& Trindade, Z. A. (2013). Adolescentes aprendizes: Aspectos da inserção profissional e mudanças na percepção de si. Revista Brasileira de Orientação Profissional, 14(1), 73-86.

Sousa, H. D., Frozzi, D., \& Bardagi, M. P. (2013). Percepção de adolescentes aprendizes sobre a experiência do primeiro emprego. Psicologia: Ciência e Profissão, 33(4), 918-933.

VandenBos, G. R. (2010). Dicionário de Psicologia da APA. Porto Alegre, RS: Artmed.

Recebido: $16 / 08 / 2017$

$1^{a}$ revisão: 20/11/2017

$2^{a}$ revisão: $11 / 12 / 2017$

Aceite final: $12 / 12 / 2017$

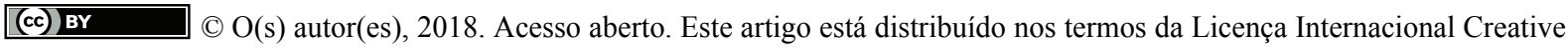
Commons Atribuição 4.0 (http://creativecommons.org/licenses/by/4.0/), que permite o uso, distribuição e reprodução sem restrições em qualquer meio, desde que você dê crédito apropriado ao(s) autor(es) original(ais) e à fonte, fornecer um link para a licença Creative Commons e indicar se as alterações foram feitas. 